\title{
GAIA Level 3b Neonatal Infectious Meningitis
}

National Cancer Institute

\section{Source}

National Cancer Institute. GAIA Level 3b Neonatal Infectious Meningitis. NCI Thesaurus. Code C127990.

GAIA Level 3b Neonatal Infectious Mening itis is defined by three criteria: first, no lumbar puncture is done or no sample is available; second, temperature greater than or equal to 37.5 degrees $C$ or less than 35.5 degrees C; third, four or more of the following requirements must be met: a) History of convulsions; b) Lethargy or irritability; c) Coma; d) Apnea; e) Bulging fontanel; f) Neck stiffness. 